\title{
Simulating Spatial and Temporal Dynamics of Soil Moisture and Gully Flow Using Improved Grid-Xinanjiang Model with a Prior Parameter Estimates
}

\author{
Bingxing Tong ${ }^{1}$, Zhijia $\mathrm{Li}^{1}$, Cheng Yao ${ }^{1}$, Moyang Liu ${ }^{2}$, and Junfu Gong ${ }^{1}$ \\ ${ }^{1}$ Hohai University \\ ${ }^{2}$ Australian National University
}

May 10, 2021

\begin{abstract}
To systematically generalize the influence of gullies on floods, a distributed model named Improved Grid-Xinanjiang (GXAJ), and a priori parameters estimation scheme based on the Global Digital Soil Mapping System (SoilGrids) are proposed. Within a watershed divided into a series of orthogonal cells, shallow furrows and trenches inside the cells are conceptualized as primary gullies, in which water movement is simulated by kinematic wave equation considering the gullies density, and well-developed grooves between cells are considered as main gullies, where water moves as a kinematic wave and enters the rivers. The simulation of 27 flood events in the Tunxi watershed of Anhui Province from 2008 to 2017 was implemented, and the simulation results were compared with that of Xinanjiang model (XAJ). The relative runoff volume error and flood peak error of the GXAJ model and XAJ model are $8.4 \%$ and $10.7 \%, 8.9 \%$ and $12.1 \%$, respectively. The GXAJ model outperforms in the simulation of flood peak, and is capable of producing the dynamics of soil water and gullies flow. The spatial characteristics and the sensitivity of parameters, free storage capacity and gullies density, at various phases, that is, initial, rise, peak, fall and tail, have been analyzed. The value of free storage capacity decreases and then increases with the increase of altitude and distance from the river. The gullies density in the bank and ridge area is greater than that in the middle of the slope segment. Sensitivity analysis shows that gullies density has the noticeable influence on the relative runoff volume error and Nash-Sutcliffe coefficient in the rise phase, while free water storage capacity has a significant effect on the relative runoff volume error during the flood rise phase and Nash-Sutcliffe coefficient in peak phase, respectively.
\end{abstract}

\section{Hosted file}

Main Document.pdf available at https://authorea.com/users/412986/articles/521464-simulatingspatial-and-temporal-dynamics-of-soil-moisture-and-gully-flow-using-improved-grid-

xinanjiang-model-with-a-prior-parameter-estimates 

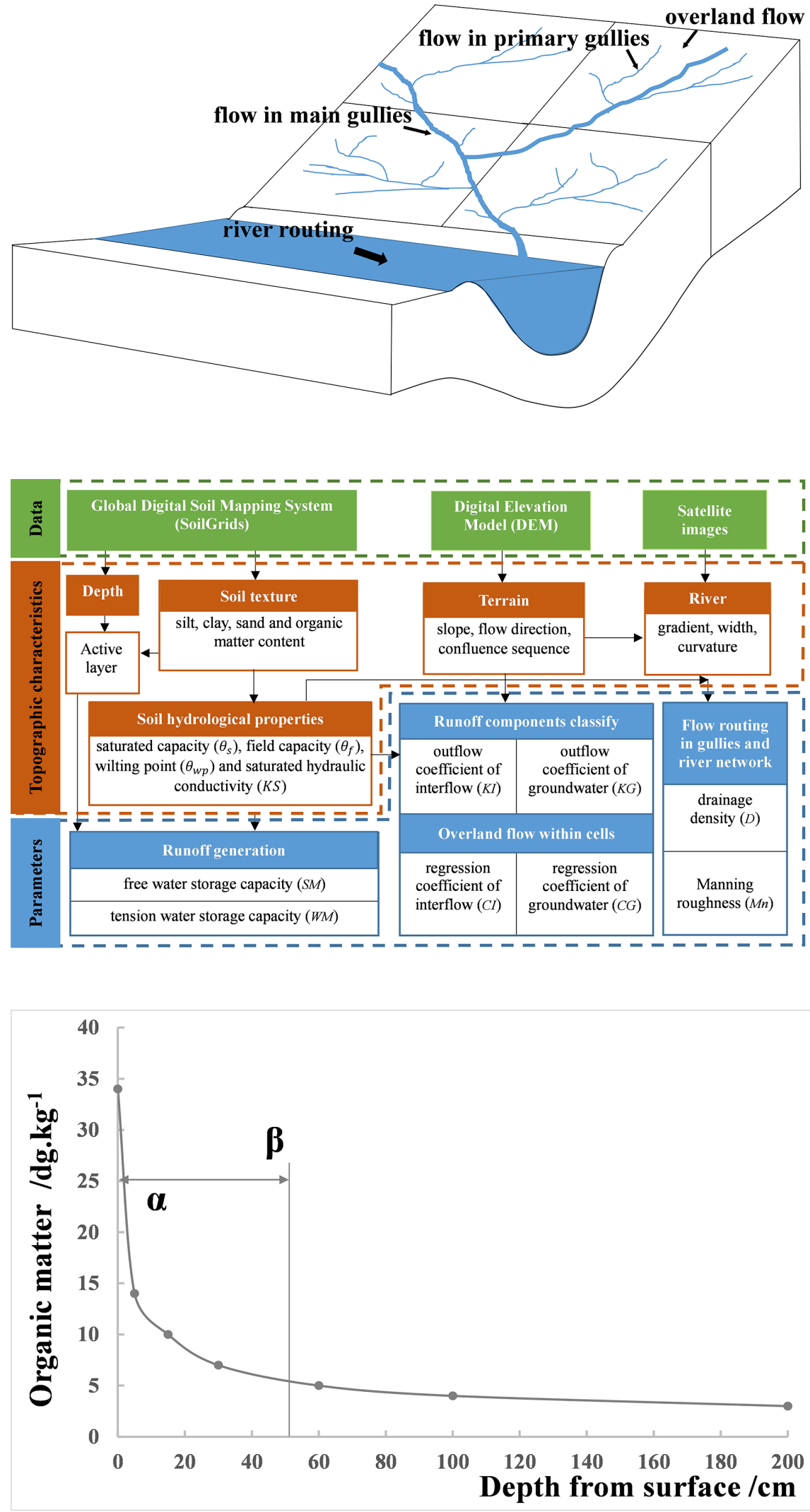

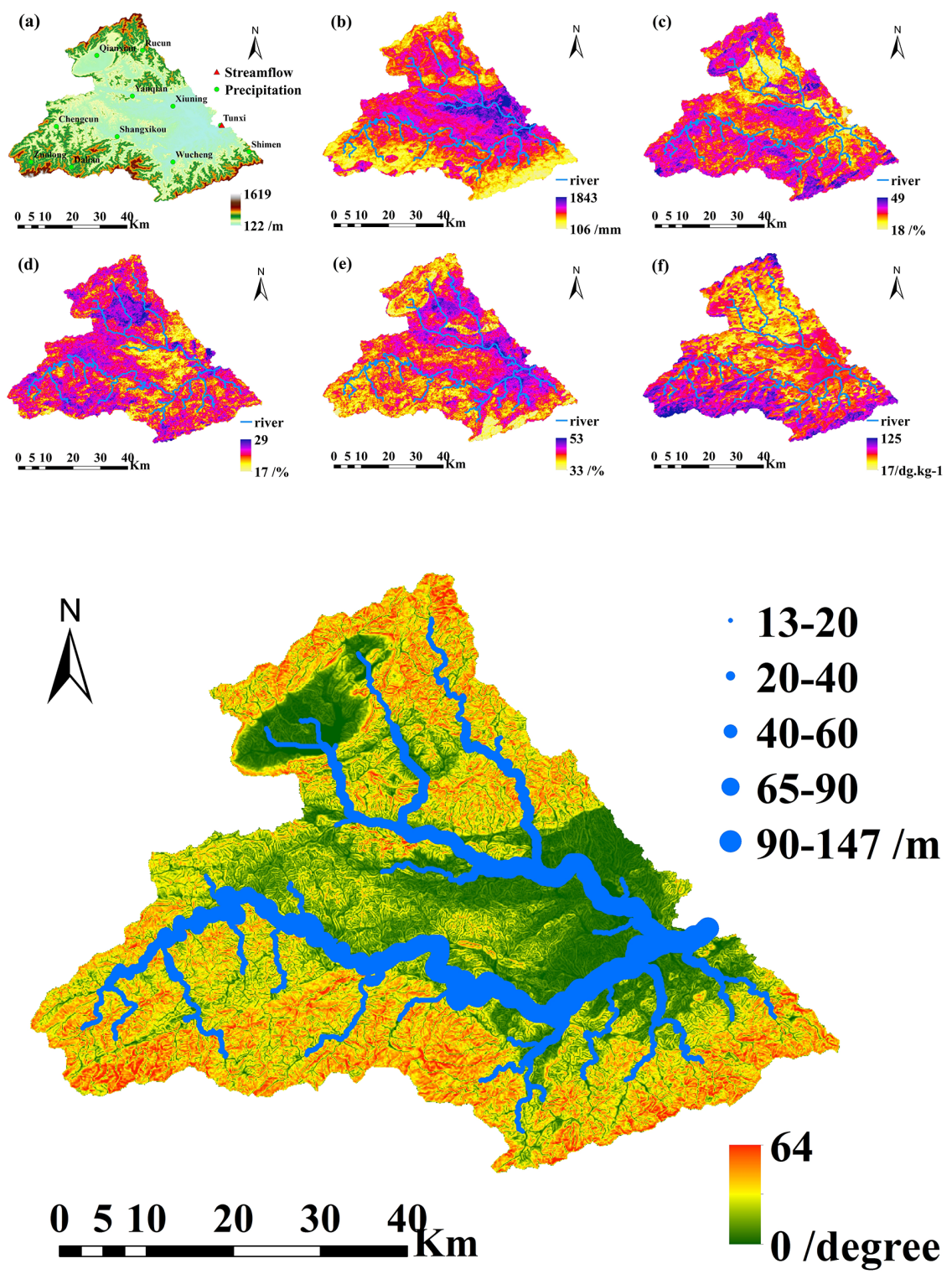

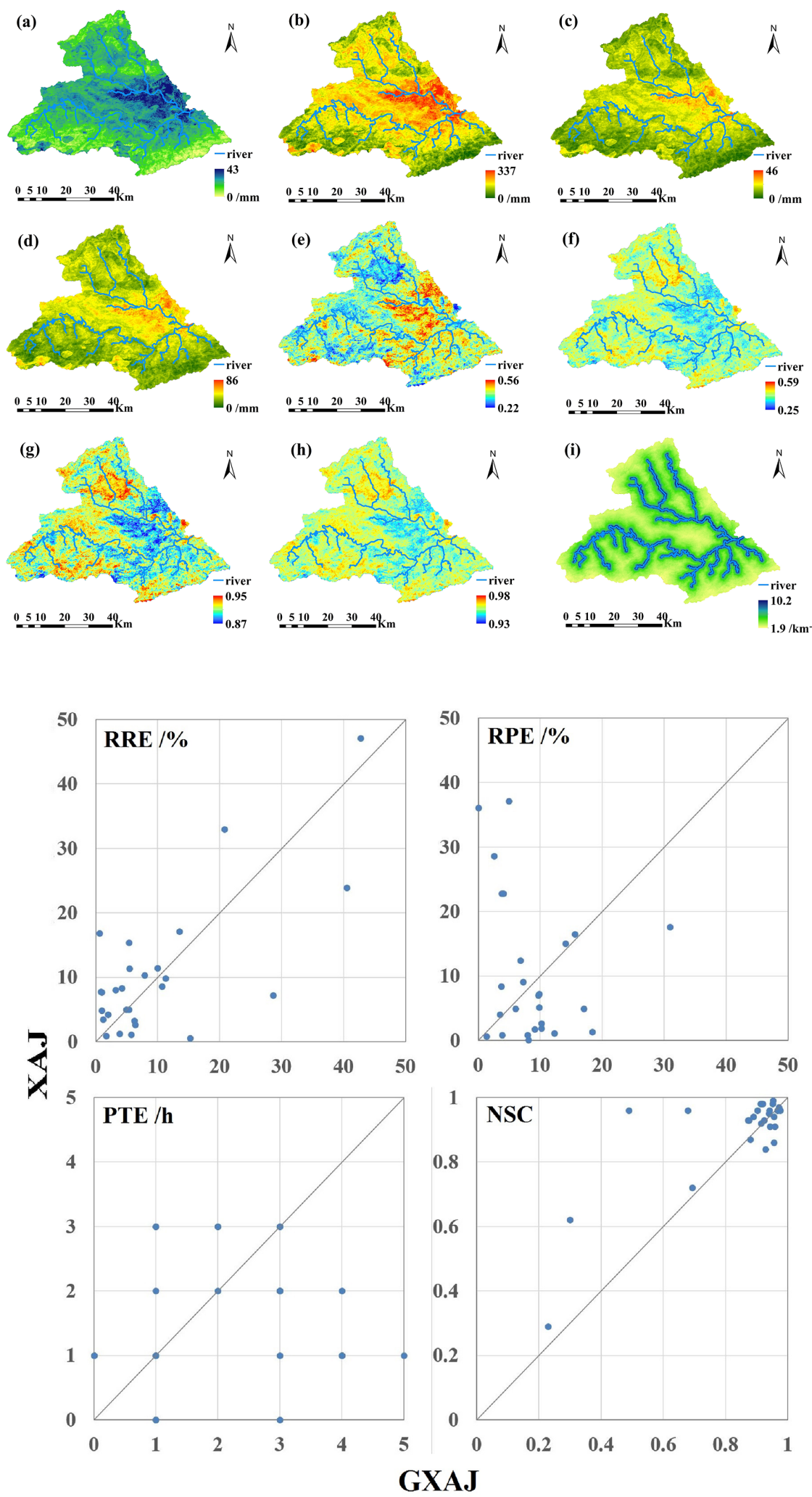

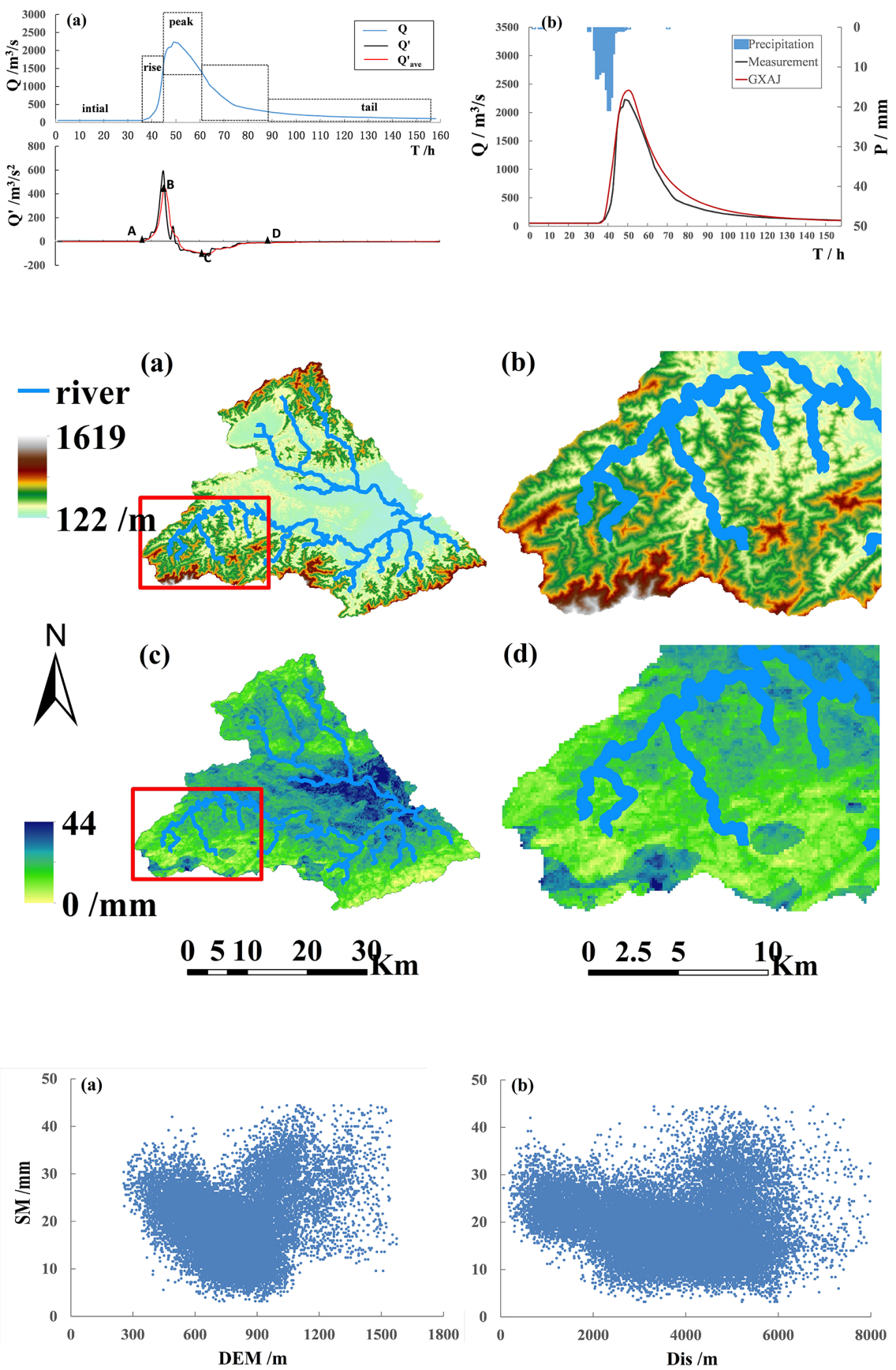

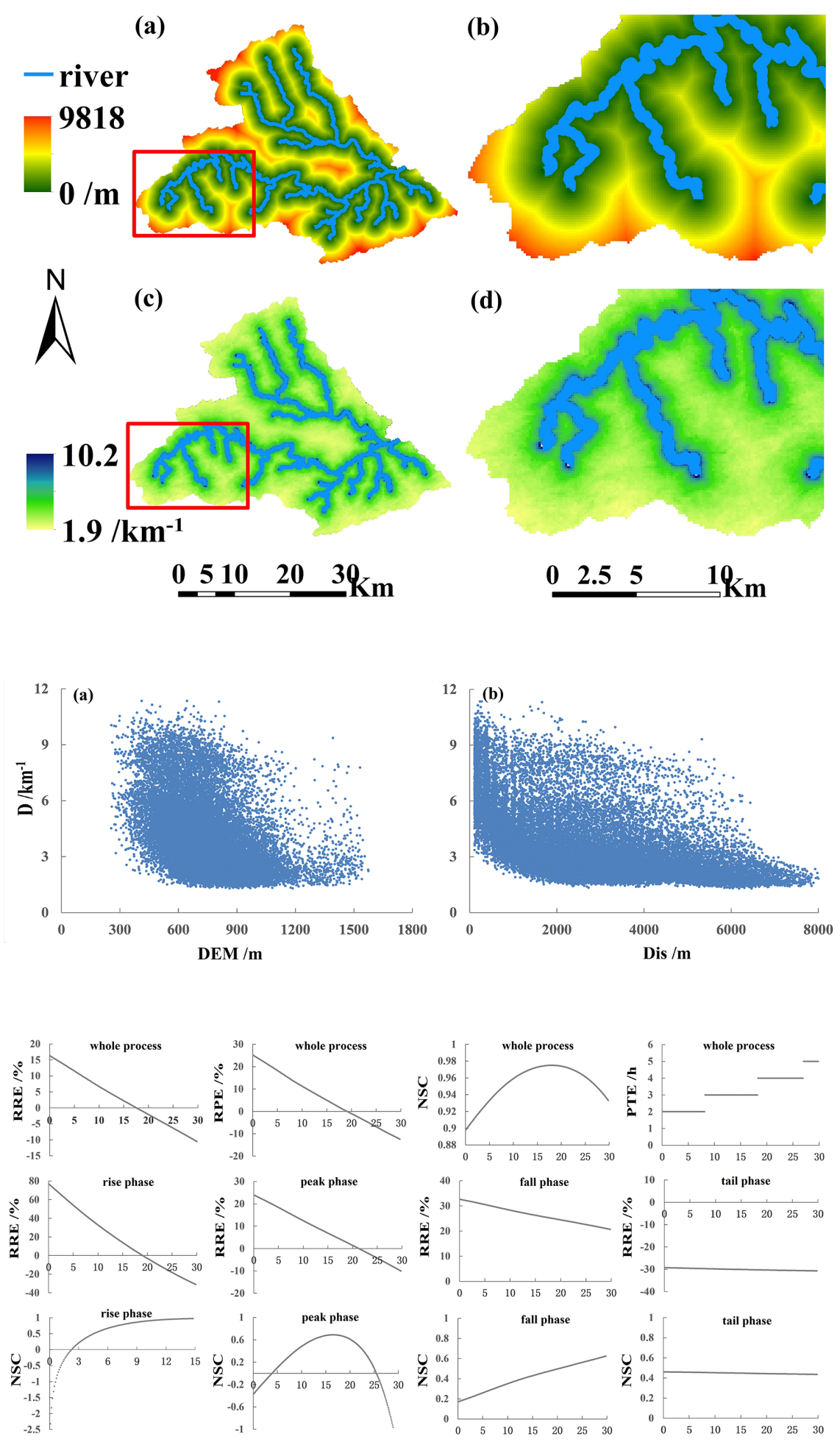

SM /mm 

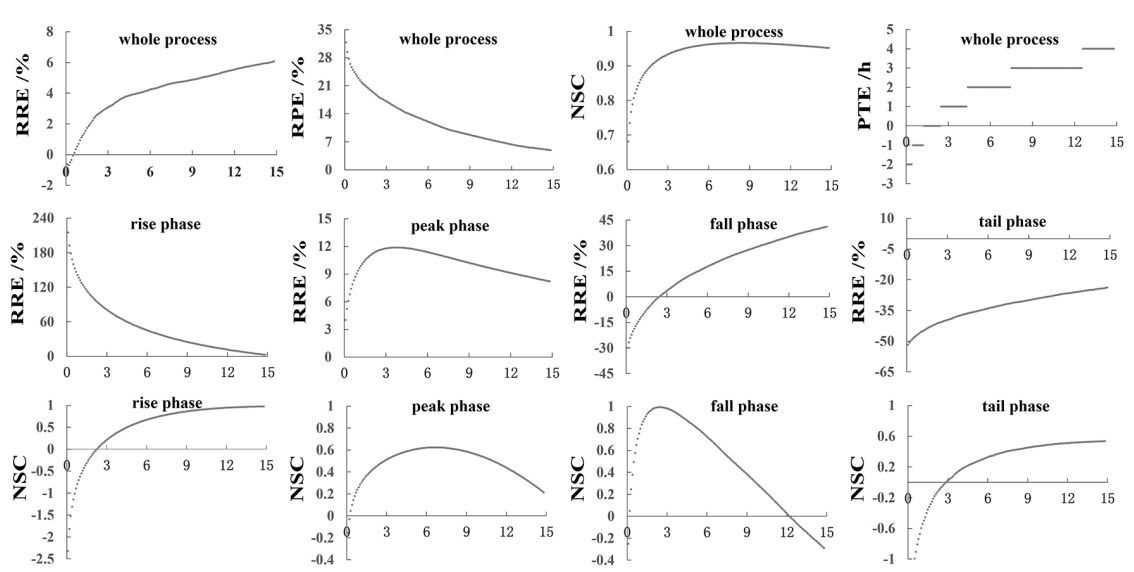

D $/ \mathbf{k m}^{-1}$
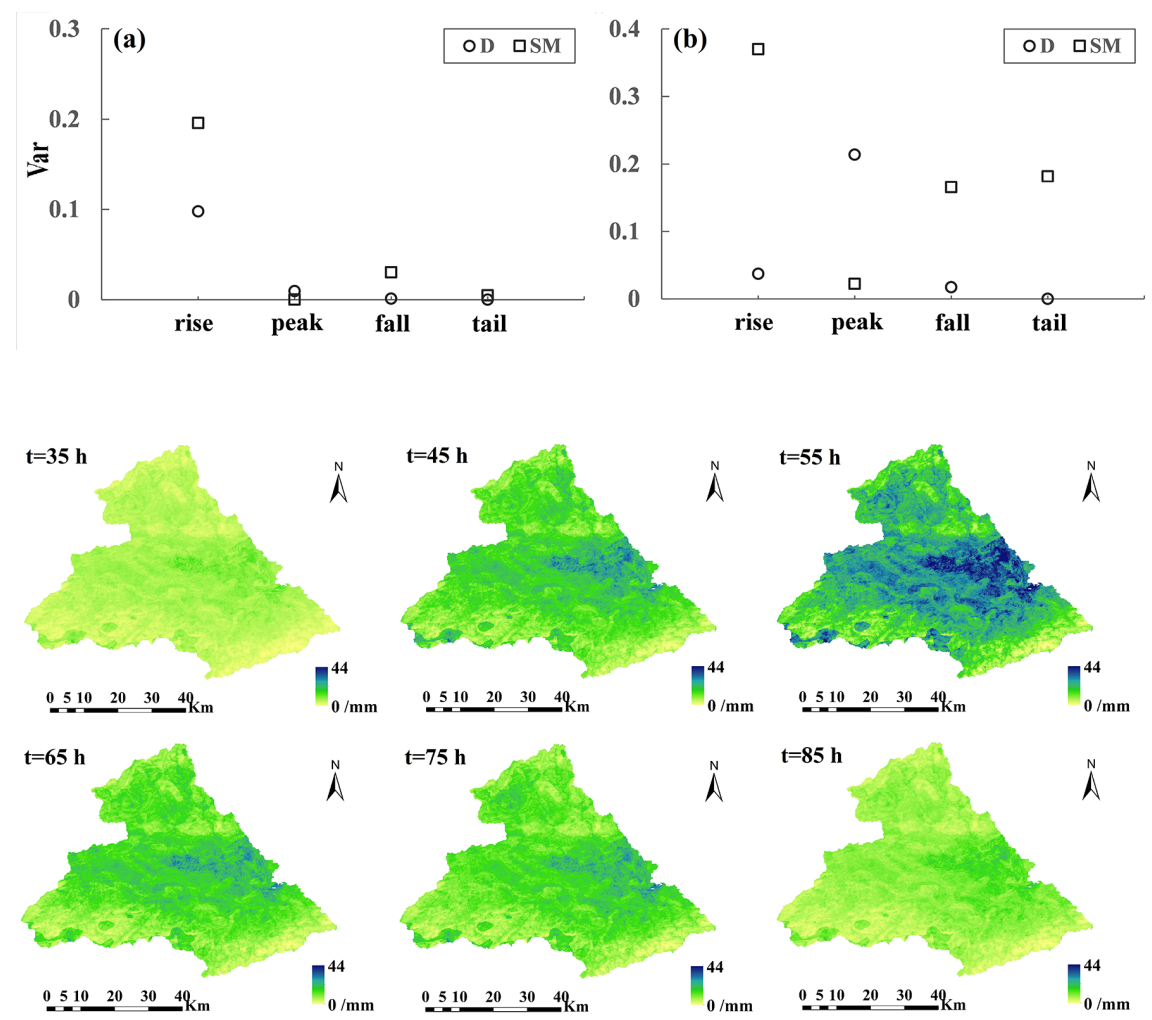


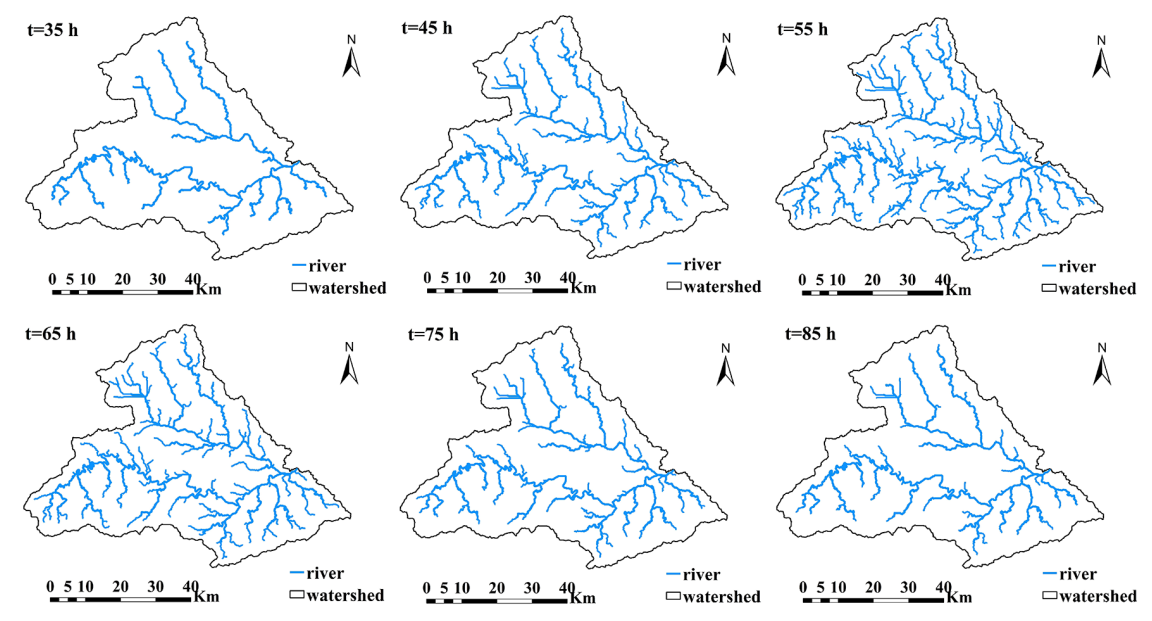

